\title{
Physiologic risk factors for early acute kidney injury in severely injured patients
}

\author{
Sklienka $\mathrm{P}^{1,2}$, Maca $\mathrm{J}^{1,2}$, Neiser $\mathbf{J}^{1,2}$, Bursa $\mathrm{F}^{1,2}$, Sevcik $\mathrm{P}^{1,2}$, Frelich $\mathrm{M}^{1,2}$, Petejova $\mathrm{N}^{3}$, Svagera $\mathrm{Z}^{4}$, \\ Tomaskova $\mathrm{H}^{5}$, Zahorec $\mathrm{R}^{6}$
}

Department of Anesthesiology and Intensive Care Medicine, University Hospital Ostrava, Ostrava, Czech Republic. peter.sklienka@seznam.cz

\begin{abstract}
BACKGROUND: The evaluation of the predictive value of the neutrophil gelatinase-associated lipocalin (NGAL) for an early acute kidney injury (AKI) development in severely injured patients. Determination of the time-dependent roles of trauma-related physiologic markers of tissue hypoxia, systemic inflammation and rhabdomyolysis in AKI development.

METHODS: 81 adult patients were screened for the presence of AKI for eight consecutive days following the injury. Arterial levels of plasma NGAL, lactate, interleukin-6, procalcitonin, and myoglobin were investigated at 24 hours (T1), 48 hours (T2), and 96 hours (T3) after the injury.

RESULTS: The incidence of AKI was $32.1 \%$. Patients with AKI were older, but no significant difference in injury severity was observed. NGAL levels were significantly higher in the AKI group at T1, T2, and T3 when compared to the non-AKI group. Lactate levels were significantly higher in the AKI group at T2 only, and IL-6 levels were significantly higher in the AKI group at T2 and T3. Procalcitonin and myoglobin levels were significantly higher in the AKI group at T1, T2, and T3, when compared to the non-AKI group. Positive correlations were found between plasma NGAL and all screened physiological factors at all defined time points. CONCLUSION: Development of AKI after blunt trauma is very complex and multifactorial. Activation of the systemic inflammatory response and rhabdomyolysis (high concentration of myoglobin) were strongly involved in AKI development. Blood NGAL levels after injury were significantly higher in patients, who developed posttraumatic AKI. Plasma NGAL, lactate, procalcitonin, interleukin-6, and myoglobin had potential to be useful parameters for risk stratification and prediction of AKI after trauma (Tab. 6, Ref. 40). Text in PDF www.elis.sk

KEY WORDS: severe injury, acute kidney injury, neutrophil gelatinase-associated lipocalin, tissue hypoxia, inflammatory response, rhabdomyolysis.
\end{abstract}

List of abbreviations: AKI - Acute Kidney Injury, KDIGO Kidney Disease: Improving Global Outcomes, NGAL - Neutrophil Gelatinase-associated Lipocalin, ISS - Injury Severity Score,

${ }^{1}$ Department of Anesthesiology and Intensive Care Medicine, University Hospital Ostrava, Ostrava, Czech Republic, ${ }^{2}$ Department of Intensive Care Medicine and Forensic Studies, Faculty of Medicine, University of Ostrava, Ostrava, Czech Republic, ${ }^{3}$ Department of Internal Medicine, University Hospital Ostrava, Ostrava, Czech Republic, ${ }^{4}$ Institute of Laboratory Diagnostics, University Hospital Ostrava, Ostrava, Czech Republic, ${ }^{5}$ Department of Epidemiology and Public Health, Faculty of Medicine, University of Ostrava, Ostrava, Czech Republic, and ${ }^{6}$ Department of Anesthesiology and Intensive Medicine. Faculty of Medicine, Comenius University, Bratislava, St. Elizabeths Cancer Institute, Bratislava, Slovakia

Address for correspondence: P Sklienka, Department of Anesthesiology and Intensive Care Medicine, University Hospital Ostrava, 17. listopadu 1790, CZ-708 52 Ostrava, Czech Republic.

Phone: +420.59 .7372713 , Fax: +420.59 .7372762$

Acknowldgement: Study supported by Ministry of Health of the Czech Republic MH CZ-DRO-FNOs/2013 grant. Dr Sklienka, Dr Petejova, Dr Svagera and Dr Tomaskova were participants of Ministry of Health of the Czech Republic MH CZ-DRO-FNOs/2013 grant "Identification of the risk factors for acute kidney injury in patients with polytrauma". Founder had no influence on the performing or publication of the study.
IL-6 - interleukin 6, PCT - procalcitonin, $\mathrm{sCr}$ - serum creatinine, MODS - Multiorgan Dysfunction Syndrome, ROC - Receiver operator characteristic, RRT - Renal Replacement Therapy, SD - standard deviation, IQR - inter-quantile range, AUROC - area under receiving operator curve, AKIN - Acute Kidney Injury Network, RIFLE - Risk, Injury, Failure, Loss of function, End stage, DAMPs - Damage-Associated Molecular Patterns, sCk - serum creatine kinase

\section{Introduction}

Trauma is the main cause of mortality in developed countries for individuals aged 1-45 years (1). Most injuries leading to death occur immediately following an accident, but late deaths are primarily associated with multiple organ dysfunction syndrome (MODS), which frequently includes acute kidney injury (AKI) (2). AKI development in injured patients is associated with adverse outcomes, surpassing other organ dysfunctions for the prediction of both multi-organ failure and death (3). In victims of trauma, mortality risk increases 1.83 times for each $1 \mathrm{mg} / \mathrm{dL}$ $(88.4 \mu \mathrm{mol} / \mathrm{L})$ elevation from the initial serum creatinine $(\mathrm{sCr})$ 


\section{$779-785$}

level, and diagnosis of renal failure increases the mortality risk 7.19 times (4).

In past, scoring systems describing severe renal dysfunction as a component of multiorgan dysfunction syndrome (MODS) have been used for AKI definition in studies examining the incidence of kidney dysfunction in severely injured patients. However, these definitions require $\mathrm{sCr}$ increase over a relatively high cut-off point ( $\mathrm{sCr}>132 \mu \mathrm{mol} / \mathrm{l}-182 \mu \mathrm{mol} / \mathrm{l}$ ), so they may underestimate significant alterations in renal function in trauma patients (5). Because even a moderate decrease in kidney function has been associated with adverse outcomes, modern criteria based on $\mathrm{sCr}$ dynamics and urine output have recently been proposed for earlier detection of acute kidney injury in critically ill patients (6). Although these definitions are more sensitive than previous criteria, both significantly elevated $\mathrm{sCr}$ and oliguria still could represent relatively late signs of serious deterioration of kidney function (7). The need for AKI identification at a stage, when functional and morphologic kidney changes are still reversible, led to the search for novel AKI biomarkers. Among these, both plasma and urine levels of neutrophil gelatinase-associated lipocalin (NGAL) offered an acceptable profile for the detection of subtle changes in kidney function and the prediction of subsequent AKI development in critically ill patients (8). The validity of urine NGAL to predict AKI development in victims of injury has been demonstrated, but data elucidating the role of the plasma NGAL for early diagnosis of posttraumatic AKI are lacking (9).

The pathophysiology of AKI in critically ill trauma patients is multifactorial, but the roles for various pathophysiologic factors associated directly with trauma have not been fully described yet. Hemorrhagic shock resulting in kidney hypoperfusion was considered the main cause of posttraumatic AKI in the past. Furthermore, severe injury causes muscle tissue destruction, leading to rhabdomyolysis and the release of myoglobin, a molecule with a direct nephrotoxic activity. Mechanisms accompanying the systemic inflammatory response to various insults have been also identified as relevant factors contributing to AKI development in critically ill, but their role in the development of posttraumatic AKI has not yet been explained in detail. Moreover, all of these injury-induced physiologic pathways are evolving early after injury and could determine kidney injury development with a various intensity during the time.

The main goal of this study was to evaluate the validity of the plasma NGAL for AKI prediction in severely injured patients. The secondary goals were to determine the time-dependent role of injury-related physiologic risk factors (tissue hypoxia, systemic inflammatory response, rhabdomyolysis) in AKI development.

\section{Methods}

The Medical Ethics Committee of the University Hospital of Ostrava approved the study (reference number: 219/b/2013IRB) on May 30, 2013, which conformed to the tenets of the Declaration of Helsinki. Each awake and conscious participant signed the Informed Consent Form approved by the Ethics Committee of the University Hospital Ostrava. Approval by two physicians not in- volved in the study was needed for the enrollment of unconscious study subjects, who were unable to sign an informed consent. In accordance with the Czech law and the Ethics Committee of the University Hospital Ostrava, no informed consent to participate was needed from their legal guardians or next of kins.

All severely injured adult patients defined by ISS $\geq 25$ were enrolled between June 2013 and December 2016 in this monocentric (Department of Anesthesiology and Critical Care of the University Hospital of Ostrava; Level 1 Trauma Center), prospective, observational study. The exclusion criteria included age $<18$ years, history of chronic kidney dysfunction, pregnancy, expected death within 24 hours of injury.

We collected basic demographic parameters including age, gender, ISS, and mechanism of injury. Laboratory parameters included arterial NGAL, lactate level, IL-6 and PCT were obtained at 24 hours (T1), 48 hours (T2), and 96 hours (T3) following injury. Serum creatinine level as measured daily at 6.00 a.m. and urine output counted hourly from the admission on ICU (Day 0) to Day 8 were the basis for AKI evaluation. Because recent pre-injury creatinine values were unknown for almost all subjects, the first serum creatinine level $(\mathrm{sCr})$ taken at admission to the emergency room was considered the baseline reference value. Patients were screened for the evidence of early AKI for eight days following injury, and patients were divided into the AKI group and nonAKI group defined by AKI development during Day 0 to Day 8 for further analysis.

According to the KDIGO criteria, AKI was defined as any of the following: $\mathrm{sCr}$ increase of $0.3 \mathrm{mg} / \mathrm{dL}$ ( $\geq 26.5 \mu \mathrm{mol} / \mathrm{L}$ ) within 48 hours, or $\mathrm{sCr}$ increase to $\geq 1.5$ times baseline (known or presumed to have occurred within the preceeding 7 days) or urine volume $<0.5 \mathrm{~mL} / \mathrm{kg} / \mathrm{h}$ for 6 hours. KDIGO criteria were also used for further AKI staging (26).

All investigated laboratory parameters were analyzed according to the manufacturer's instructions provided in the respective assay kits. Arterial NGAL, lactate and sCr concentration were determined by AU 5811 analyzer (Beckman Coulter, Inc., Brea, CA). IL-6 concentrations were assayed by solid-phase, two-site chemiluminescent enzyme immunoassay (Immulite 2000XPi, Siemens Medical Solutions Diagnostics, NY, USA), which detects levels up to $1000 \mathrm{ng} / \mathrm{L}$. Levels greater than $1000 \mathrm{ng} / \mathrm{L}$ were reported as such. Procalcitonin was determined by CentaurXP instrument (Siemens Medical Solutions Diagnostics, NY, USA). Myoglobin concentrations were determined by a solid-phase, two-site chemiluminescent immunoanalytic assay (Unicel Dxi 800, Beckman Coulter, Inc., Brea, CA)

Tab. 1. Mechanism of injury.

\begin{tabular}{lcc}
\hline Mechanism of injury & Number & $\%$ \\
\hline Vehicle accident & 34 & 42.0 \\
Pedestrian & 20 & 24.7 \\
Fall & 22 & 27.2 \\
Explosion & 2 & 2.45 \\
Cave-in at coal mine & 2 & 2.45 \\
Unknown & 1 & 1.20 \\
\hline Total & 81 & 100.00 \\
\hline
\end{tabular}


Tab. 2. Differences for myoglobin, lactate, interleukin-6 and procalcitonin between non-AKI and AKI groups.

\begin{tabular}{lccc}
\hline Variable & $\begin{array}{c}\text { Non-AKI group } \\
\text { Median (IQR 25\%-75\%) }\end{array}$ & $\begin{array}{c}\text { AKI group } \\
\text { Median }(\mathrm{IQR} 25 \%-75 \%)\end{array}$ & $\mathrm{p}$ \\
\hline Myoglobin $(\mu \mathrm{g} / \mathrm{L})$ & & & \\
T1 & $609.0(346.0-1251.0)$ & $2552.0(940.9-4419.8)$ & $<0.001$ \\
T2 & $286.0(153.0-673,1)$ & $1162.9(594.6-3104.6)$ & $<0.001$ \\
T3 & $177.5(88.4-345.3)$ & $425.8(157.7-861.8)$ & 0.002 \\
\hline Lactate $(\mathrm{mmol} / \mathrm{L})$ & & & \\
T1 & $1.89(1.28-2.36)$ & $1.96(1.39-2.76)$ & 0.379 \\
T2 & $1.30(0.98-1.90)$ & $1.76(1.39-2.37)$ & 0.005 \\
T3 & $1.28(0.84-1.63)$ & $1.56(1.17-1.88)$ & 0.065 \\
\hline IL-6 $(\mathrm{ng} / \mathrm{L})$ & & & \\
T1 & $238.0(124.0-525.0)$ & $445.5(147.0-721.0)$ & 0.176 \\
T2 & $126.0(53.2-303.0)$ & $210.5(111.3-581.5)$ & 0.028 \\
T3 & $48.8(27.2-90.0)$ & $89.4(48.6-167.3)$ & 0.027 \\
PCT $(\mu \mathrm{g} / \mathrm{L})$ & $1.15(0.59-5.11)$ & $3.89(1.19-10.65)$ & 0.017 \\
T1 & $0.88(0.47-3.00)$ & $2.38(0.79-8.50)$ & 0.008 \\
T2 & $0.50(0.22-1.10)$ & $1.69(0.47-5.20)$ & 0.001 \\
T3 &
\end{tabular}

AKI - acute kidney injury; SD - standard deviation; IQR - inter-quantile range; IL-6 - interleukin 6; PCT procalcitonin.

Tab. 3. Plasma NGAL in the non-AKI and AKI groups.

\begin{tabular}{lccc}
\hline NGAL $(\mathrm{ng} / \mathrm{mL})$ & Non-AKI group & AKI group & $\mathrm{p}$ \\
\hline T1 & Median (IQR 25\%-75\%) & Median (IQR 25\%-75\%) & \\
T2 & $110.1(78.8-174.0)$ & $216.0(147.0-396.5)$ & $<0.001$ \\
T3 & $75.5(44.3-125.0)$ & $202.9(114.0-311.0)$ & $<0.001$ \\
\hline
\end{tabular}

$\mathrm{NGAL}$ - neutrophil gelatinase-associated lipocalin; AKI - acute kidney injury; IQR - inter-quantile range.

Tab. 4. Correlation between NGAL and AKI severity (defined by KDIGO stage).

\begin{tabular}{cccc}
\hline & KDIGO stage (n) & $\begin{array}{c}\text { Median NGAL }(\mathrm{ng} / \mathrm{mL}) \\
(\mathrm{IQR} 25 \%-75 \%)\end{array}$ & $\mathrm{r}_{\mathrm{s}}$ \\
\hline \multirow{4}{*}{ T1 } & non-AKI (55) & $110.1(82.3-174.0)$ & \\
& $1(11)$ & $147.1(149.5-304.4)$ & $0.435^{*}$ \\
& $2(7)$ & $155.0(122.0-421.8)$ & \\
& $3(8)$ & $454.7(200.6-607.3)$ & \\
T2 & non-AKI 0 (55) & $78.5(49.3-125.0)$ & \\
& $1(11)$ & $120.0(91.0-204.0)$ & $0.523^{*}$ \\
& $2(7)$ & $148.0(84.0-306.0)$ & \\
& $3(8)$ & $498.5(253.3-583.1)$ & \\
T3 & non-AKI 0 (55) & $69.3(43.9-122.7)$ & \\
& $1(11)$ & $85.3(37.0-163.0)$ & $0.377^{*}$ \\
& $2(7)$ & $159.6(57.0-231.8)$ & \\
\hline
\end{tabular}

NGAL - neutrophil gelatinase-associated lipocalin; AKI - acute kidney injury; KDI$\mathrm{GO}$ - Kidney Disease: Improving Global Outcomes; $\mathrm{r}_{\mathrm{s}}-$ Spearman's coefficient; $* \mathrm{p}<0.05$.

Tab. 5. Plasma NGAL in the patients with and without indication for renal replacement therapy.

\begin{tabular}{lccc}
\hline & RRT (n) & $\begin{array}{c}\text { Median NGAL }(\mathrm{ng} / \mathrm{mL}) \\
(\mathrm{IQR} 25 \%-75 \%)\end{array}$ & $\mathrm{p}$ \\
\hline \multirow{2}{*}{ T1 } & No (76) & $125.3(84.8-208.3)$ & $\mathrm{p}=0.008$ \\
\hline \multirow{2}{*}{ T2 } & Yes (5) & $379.5(238.9-548.9)$ & No (76) \\
\hline \multirow{2}{*}{ T3 } & Yes (5) & $52.5(61.8-155.5)$ & $\mathrm{p}=0.002$ \\
\hline
\end{tabular}

NGAL - neutrophil gelatinase-associated lipocalin; RRT - renal replacement therapy; $\mathrm{IQR}$ - inter-quantile range.
The authors used Stata software version 13 for all statistical analyses. A ShapiroWilks test was used for testing the normality of the distribution. Normally or near normally distributed variables were reported as the means with standard deviations (SD) and compared by Student's t-tests. Nonnormally distributed continuous data were reported as the medians with inter-quartile ranges (IQR) and compared by Mann-Whitney U tests or Kruskal-Wallis tests. Pearson's chi-square test, Spearman's rank-order correlation, or Fisher's exact test were used to analyze categorical data as appropriate. $p$ value of $<0.05$ was considered statistically significant for all comparisons. Receiver operator characteristic (ROC) curves and areas under the curve (AUC) were determined to evaluate the accuracy of NGAL for AKI prediction. Youden's index was used for the determination of NGAL cut-off points.

\section{Results}

Demographic data. 82 consecutive patients with ISS $\geq 25$ fulfilled the enrollment criteria for the study. One patient was excluded due to technical problems (inability to measure NGAL at T1 and T2), therefore 81 patients (68 males and 13 females) were included in the analysis. The mean age of the population was $43.6 \pm 15.9$ years and the mean ISS was 32.8 \pm 7.6. There were no significant differences between genders in age (males $43.0 \pm 15.7$ years, females $46.9 \pm 16.9$ years; $p=$ 0.448 ) or ISS (males $32.5 \pm 7.5$, females $34.3 \pm 8.5$; $\mathrm{p}=0.416$ ). All patients with ISS $\geq 25$ during the study period suffered from blunt injury (Tab. 1).

Incidence and risk factors of AKI. Crude AKI incidence was $32.1 \%(\mathrm{n}=26)$. Eleven patients $(13.6 \%)$ developed KDIGO stage 1 acute kidney injury, seven patients $(8.6 \%)$ developed KDIGO stage 2, and eight patients (9.9\%) developed KDIGO stage 3. Five $(6.2 \%)$ subjects required renal replacement therapy (RRT), and all were classified as KDIGO stage 3 at the time of RRT initiation. Patients with AKI were significantly older than patients without AKI (non-AKI group $40.3 \pm 14.3$, AKI group $50.6 \pm 17.1$; $\mathrm{p}=0.006)$. There was no significant difference in the mean ISS between the groups (non-AKI group $32.6 \pm 7.1$, AKI group 33.2 $\pm 8.9 ; \mathrm{p}=0.733)$, and there was no difference in AKI incidence between the genders (males $30.9 \%(21 / 68)$, females $38.5 \%$ $(5 / 13) ; \mathrm{p}=0.706)$.

Trauma-related physiological risk factors. Lactate levels in AKI group differed significantly at T2, but neither at T1 nor T3. The values of IL-6 were significantly higher in AKI group at T2 and T3 but not at T1. Procalcitonin levels were significantly higher in AKI group at all defined time points. Myoglobin levels were significantly higher in AKI group at all time points too (Tab. 2). 
Tab. 6. Correlation of NGAL

\begin{tabular}{lcccc}
\hline \multirow{2}{*}{ Time/variable } & \multicolumn{4}{c}{$\mathrm{r}_{\mathrm{s}}\left(\right.$ for $\left.\mathrm{NGAL}_{\max }\right)$} \\
\cline { 2 - 5 } & Lactate & $\mathrm{IL}-6$ & $\mathrm{PCT}$ & Myoglobin \\
\hline T1 & $0.2998^{*}$ & $0.4789^{*}$ & $0.5100^{*}$ & $0.2484^{*}$ \\
T2 & $0.3657^{*}$ & $0.3772^{*}$ & $0.5365^{*}$ & $0.2796^{*}$ \\
T3 & $0.3821^{*}$ & $0.2406^{*}$ & $0.5992^{*}$ & $0.2363^{*}$ \\
Maximal & $0.3486^{*}$ & $0.4909^{*}$ & $0.5486^{*}$ & $0.2644^{*}$
\end{tabular}

NGAL - neutrophil gelatinase-associated lipocalin; IL-6 - interleukin 6; PCT - procalcitonin; $r_{\mathrm{s}}-$ Spearman's coefficient; $* \mathrm{p}<0.05$.

NGAL and AKI. NGAL plasma levels were significantly higher in AKI group at all defined time points and at maximal recorded level during the first 96 hours following injury (Tab. 3).

Cut-off values of NGAL for AKI development were 149.5 $\mathrm{ng} / \mathrm{mL}$ at $\mathrm{T} 1$ with area under receiving operator curve (AUROC) 0.693 , sensitivity $73.1 \%$ (95\% (CI) $52.0 \%-87.7 \%$ ), specifity $65.5 \%(95 \%$ (CI) $51.3 \%-77.4 \%$ ), positive predictive value 50.0 $\%$ (95\% (CI) $33.7 \%-66.3 \%$ ), negative predictive value $83.7 \%$ (95\% (CI) $68.7 \%-92.7 \%$ ). Cut-off values of NGAL for AKI development were $111.0 \mathrm{ng} / \mathrm{mL}$ at T2 with AUROC 0.73, sensitivity $76.9 \%$ (95\% (CI) $55.9 \%-90.2 \%$ ), specifity $69.1 \%(95 \%$ (CI) $55.0 \%-80.5 \%$ ), positive predictive value $54.1 \%$ (95\% (CI) $37.1 \%-70.5 \%$ ), negative predictive value $86.4 \%$ (95\% (CI) 72.0 $\%-94.3 \%$ ). Cut-off values of NGAL for AKI development were d $154.6 \mathrm{ng} / \mathrm{mL}$ at T3 with AUROC 0.73, sensitivity $57.7 \%$ (95\% (CI) $37.2 \%-76.0 \%$ ), specifity $87.3 \%$ (95\% (CI) $74.9 \%-94.3$ $\%$ ), positive predictive value $68.2 \%$ (95\% (CI) $45.1 \%-85.3 \%$ ), negative predictive value $81.4 \%$ (95 \% (CI) $68.7 \%-89.9 \%$ ).

Weak to moderate correlations were found between NGAL and AKI severity at all defined time points (Tab. 4). Plasma NGAL levels were significantly higher in patients, who subsequently required RRT at all defined time points too (Tab. 5).

Correlations between NGAL and trauma-related physiologic risk factors. A weak, but significant positive correlation $\left(r_{\mathrm{s}}=0.224\right.$; $p=0.045$ ) was found between ISS and maximal recorded level of NGAL $\left(\mathrm{NGAL}_{\text {max }}\right)$ the first 96 hours after injury Significant weak to moderate positive correlations were found between $\mathrm{NGAL}_{\text {max }}$ and all screened laboratory parameters at all defined time points too (Tab. 6).

\section{Discussion}

Acute kidney injury as defined by KDIGO occurs in more than $50 \%$ of all critically ill patients, and AKI development is associated with adverse outcomes including an increased mortality (10). Severe injury is considered a significant risk factor in predictive models for AKI development in critically ill patients (11). In our study, the incidence of early AKI as defined by KDIGO criteria was $32.1 \%$ in the patients with ISS $\geq 25$. This is in consent with papers reporting AKI incidence defined by currently generally accepted criteria (AKIN, RIFLE, and KDIGO) in the range 15-74.2 $\%$ in severely injured patients (12).

Urine output and serum creatinine levels represented the basis for all AKIN, RIFLE, and KDIGO criteria. However, both oliguria and $\mathrm{sCr}$ increase could represent relatively late signs of an already serious deterioration of kidney function. Current research in AKI diagnostics is focused on the identification of clinical and laboratory biomarkers correlating with AKI development during the earliest possible time window before the onset of oliguria and $\mathrm{sCr}$ elevation. Among a wide range of reported AKI biomarkers, NGAL levels in both plasma and urine offered an acceptable profile for the detection of subtle changes in kidney function and the prediction of subsequent AKI development, and NGAL plasma levels between $97-150 \mathrm{ng} / \mathrm{mL}$ were found to be the predictive cut-off values for AKI development in critically ill patients (13). In victims of trauma, elevated plasma levels of NGAL were associated with a risk of the development of posttraumatic multiorgan dysfunction syndrome (14). Plasma levels of NGAL were superior to traditional AKI biomarkers such as: creatinine and UOP in AKI prediction both in burn and non-burned trauma patients and NGAL-based algorithm helped predict AKI 61.8 (32.5) hours faster than the Kidney Disease and Improving Global Disease Outcomes (KDIGO) criteria (15). In victims of trauma, urinary levels of AKI biomarkers (including NGAL) were associated with the injury severity, and early urinary NGAL levels correlated with subsequent AKI development, a need for renal replacement therapy, and mortality $(9,16,17)$. Because urine NGAL monitoring is problematic in patients with oliguria or direct injury of the urine tract, we investigated plasma NGAL levels only. We found a significant difference between the $\mathrm{AKI}$ and non-AKI groups at all defined time points as well as in NGALmax value during the first 96 hours after injury. The plasma NGAL cut-off values for AKI prediction identified in our study (149.5 ng/mL, $111.0 \mathrm{ng} / \mathrm{mL}$, and $154.6 \mathrm{ng} / \mathrm{mL}$ at T1, T2, and T3, respectively) correlated well with the plasma NGAL cut-off values defined for the mixed groups of patients in studies reported above. Moreover, we showed the correlation of plasma NGAL levels at all defined time points with AKI severity (defined by KDIGO) as well as with subsequent requirement of renal replacement therapy. These results are in accordance with papers reporting the concept of "subclinical AKI", where patients without AKI, but presenting elevated NGAL levels reached a higher mortality and RRT needing in comparison to patients with normal NGAL $(18,19)$. Based on our results we suggest that plasma NGAL level monitoring could be a useful additional tool in both diagnostics of early AKI and a prediction of RRT necessity in critically ill trauma patients.

Although injury severity represents the crucial factor for the development of posttraumatic complications, the correlation between the injury severity and AKI development varied in previous reports (5). In the retrospective analysis of the data of 78,345 adult trauma patients, the incidence of severe AKI requiring renal replacement therapy was $0.5 \%$ for all the patients admitted to the hospital, but $8.3 \%$ in a subpopulation of severely injured patients requiring an admission to the intensive care unit (ICU) (20). We screened injured patients with a median ISS of 29 (mean: 32.8), which is higher than the ISS reported in most studies mentioned above. We did not find the correlation between the injury severity 
as defined by ISS and AKI development. However, the correlation between ISS and AKI could be hidden in our study due to the relative homogeneity in our population, since ISS of 25 represents the cut-off point for predicting major complications and mortality in injured patients $(21,22)$. ISS level of 25 was recognized as a significant cut-off point for AKI development in the subpopulation of injured patients too $(9,16)$. Moreover, all the patients in our study suffered from a blunt injury, which was established as an independent risk factor for AKI development in the victims of injury (23). At the last, ISS is based strictly on the degree of anatomic injury, but does not contain relevant physiologic factors contributing to AKI development in the victims of trauma. Combined scoring systems, which include both anatomic and physiologic variables indicated a higher efficiency of these systems in predicting mortality compared to ISS (24). The positive correlation between NGALmax and ISS without a difference in ISS between the AKI and non-AKI groups could be explained by the fact that the injury severity represents a significant insult for kidney function, but other factors resulting from the injury are involved in the development of clinically significant AKI too.

Severe trauma is frequently associated with tissue hypoxia resulting from trauma-related circulatory shock. According to previous reports, trauma patients, who repaid oxygen debt within the first 24 hours had a lower morbidity and mortality, and blood lactate levels represented a marker of severity and serious clinical course $(25,26)$. Previous reports of the correlation between shock parameters and AKI incidence are heterogeneous because different criteria (systolic blood pressure, mean arterial pressure, base deficit, serum lactate, amount of applied transfusion, vasoactive drug administration, and many other parameters) were used as the criteria for the shock. Serum lactate is the most relevant marker of tissue hypoxia resulting from the circulatory shock, and serum lactate dynamics were considered as reliable end-points for the efficacy of hemodynamic resuscitation in the prevention of early multiorgan dysfunction syndrome (27). We found a significant difference in arterial lactate between the AKI group and the non-AKI group at $\mathrm{T} 2$, but not at $\mathrm{T} 1$ or $\mathrm{T} 3$. These results are in contradiction with the traditional concept of hypoxia-induced etiology of acute kidney injury in the victims of severe injury. However, lactate levels were relatively low in our study as early as 24 hours after injury due to the routine application of the therapeutic protocols aimed at early hemodynamic stabilization, hemostatic resuscitation, and the early elimination of oxygen debt in our critically injured patients. These results indicate that current evidence-based resuscitation protocols could reduce tissue hypoxia-related risk of acute kidney injury in the victims of trauma.

Pathways related to systemic inflammation are involved in AKI pathophysiology in critically ill (28). Severe trauma induces the activation of the innate immune response, primarily through the release of Damage-Associated Molecular Patterns (DAMPs; alarmins) from injured tissue (29). Although the pro-inflammatory and anti-inflammatory immune response develops simultaneously immediately after the injury, an excessive pro-inflammatory response is a hallmark of the early post-injury organ dysfunctions, while a later overwhelming anti-inflammatory response is crucial for late organ dysfunction typically resulting from a suppressed host immunity and recurrent infections $(2,30)$. Excessive activation of the pro-inflammatory pathway during the first 24 hours of injury has been reported as a predictive factor for AKI development too (31). We investigated the correlation between AKI development and arterial levels of the early pro-inflammatory cytokines interleukin-6 and procalcitonin. IL-6 levels early after injury correlated with the degree of anatomic injury as defined by ISS and predict subsequent complications including multiple organ dysfunction syndromes (including AKI) and mortality in previous reports (32, 33). In our study, IL-6 was significantly higher in the AKI-group at $\mathrm{T} 2$ and $\mathrm{T} 3$ but not at T1. PCT is a marker of bacterial infection, but it is also rapidly elevated in various non-infectious forms of systemic inflammation including injury. Elevated PCT levels immediately after trauma are predictive of both infectious and noninfectious complications and correlate with the development of organ dysfunctions and increased mortality $(34,35)$. We found a significant difference between the AKI-group and non-AKI group in serum PCT at all of T1, T2, and T3. These results suggest that while the activation of the innate immune response immediately after trauma represents a physiological response to severe injury, the excessive or persistent activation of the systemic inflammatory pathway are relevant risk factors for the development of AKI.

Rhabdomyolysis is a clinical condition characterized by damage of skeletal muscle mass with a subsequent release of myoglobin to the extracellular fluid. Circulating myoglobin causes renal vasoconstriction, induces membrane lipide peroxidation in kidney tubular cells, and forms intraluminal precipitates in proximal tubules. Severe injury causes a varying degree of rhabdomyolysis in almost $85 \%$ of the patients and rhabdomyolysis increases the risk of AKI development 4.67 times $(36,37)$. Interestingly, rhabdomyolysis-induced AKI occurs less frequently in combination with other organ dysfunctions and is associated with better outcomes than AKI induced predominantly by other causes (23). The diagnosis of rhabdomyolysis is based on laboratory findings of elevated serum levels of both creatine kinase (sCk) and myoglobin. We investigated serum levels of myoglobin only because myoglobin exerts a direct nephrotoxic effect, and serum myoglobin levels were reported to be superior to sCK for AKI prediction (38, 39). Moreover, peak sCk occurs relatively late after injury (40). We found significantly higher serum myoglobin levels in the AKIgroup at all investigated periods. Thus, rhabdomyolysis defined by elevated serum myoglobin levels during the first 96 hours after injury represented a significant risk factor for posttraumatic AKI.

Various predictive factors, models, and equations have been reported to predict posttraumatic AKI (5). However, data were collected mostly at the time of admission to the emergency department or ICU in most clinical studies evaluating risk factors for posttraumatic AKI. To our best knowledge, our paper represents the first study reporting the time-dependent impact of relevant physiologic factors on AKI development in the victims of severe injury as well as their correlations with a biomarker of kidney injury. We found a weak to moderate significant correlations between NGALmax and all screened laboratory parameters (arterial lactate, IL-6, PCT, myoglobin) at all defined time points. Although we did not 


\section{9-785}

find a correlation between ISS and AKI, we did find a weak, but significant positive correlation between ISS and NGALmax. Our results suggest that the development of posttraumatic AKI could not be viewed as a "single-shot" event emerging at the time of injury, but rather as a continuous process involving various risks with varying intensity over the course of illness.

We are aware of several limitations of this study. First, the number of subjects included in the study does not allow us to identify independent predictors of AKI at different time points. We also did not investigate the influence of various other factors affecting kidney function (such as the application of intravenous contrast agents, application of starches, amount of transfusion applied, number and type of surgery, intraabdominal hypertension, serum chloride level, coincidence with additional organ dysfunctions, and many others). Third, the time intervals for scheduled blood sampling may not sufficiently reflect the exact dynamics of the blood concentrations of NGAL and the other screened factors. Despite the limitations mentioned above, we believe that this paper provides notable data in the field of the pathophysiology of AKI development in severely injured patients and could serve as a basis for further research.

\section{Conclusion}

Early acute kidney injury occurs frequently in severely injured patients. Development of AKI after blunt trauma is a very complex and multifactorial pathophysiological process. Many factors are involved in the development of posttraumatic AKI including older age, excessive or prolonged activation of the systemic inflammatory response, and rhabdomyolysis. The levels of the plasma NGAL in the first 96 hours after the injury are significantly higher in patients, who develop AKI and correlate with subsequent AKI severity and renal replacement need. The results of our study confirmed the validity of plasma NGAL for AKI prediction, but also the key role of other risk factors, including blood lactate, procalcitonin, interleukin-6, and myoglobin. All these parameters may be useful for risk stratification, and prediction of AKI after blunt trauma in the critical care.

Acute kidney injury in the victims of trauma represents a continuous process involving various risks with varying intensity over the course of illness.

\section{References}

1. Bardes JM, Inaba $\mathbf{K}$, Schellenberg $\mathbf{M}$ et al. The contemporary timing of trauma deaths. J Trauma Acute Care Surg 2018; 84 (6): 893-899.

2. Sauaia A, Moore EE, Johnson JL et al. Temporal trends of postinjury multiple-organ failure: still resource intensive, morbid, and lethal. J Trauma Acute Care Surg 2014; 76 (3): 582-592.

3. Wohlauer MV, Sauaia A, Moore EE, Burlew CC, Banerjee A, Johnson J. Acute kidney injury and posttrauma multiple organ failure: The canary in the coal mine. J Trauma Acute Care Surg 2012; 72 (2): 373-378.

4. Brandt MM, Falvo AJ, Rubinfeld IS, Blyden D, Durrani NK, Horst HM. Renal dysfunction in trauma: even a little costs a lot. J Trauma 2007; 62: $1362-1364$.
5. Harrois A, Libert N, Duranteau J. Acute kidney injury in trauma patients. Curr Opin Crit Care 2017; 23 (6): 447-456.

6. Rossaint J, Zarbock A. Acute kidney injury: definition, diagnosis and epidemiology. Minerva Urol Nefrol 2016; 68 (1): 49-57.

7. Prowle JR. Acute kidney injury: Creatinine and AKI - through a glass, darkly. Nat Rev Nephrol 2013; 9 (4): 193-195.

8. Haase-Fielitz A, Haase M, Devarajan P. Neutrophil gelatinase-associated lipocalin as a biomarker of acute kidney injury: a critical evaluation of current status. Ann Clin Biochem 2014; 51 (0 3): 335-351.

9. Makris K, Markou N, Evodia E et al. Urinary neutrophil gelatinaseassociated lipocalin (NGAL) as an early marker of acute kidney injury in critically ill multiple trauma patients. Clin Chem Labor Med 2009; 47 (1): 79-82.

10. Hoste EAJ, Bagshaw SM, Bellomo R et al. Epidemiology of acute kidney injury in critically ill patients: the multinational AKI-EPI study. Intensive Care Med 2015; 41 (8): 1411-1423.

11. Kellum JA, Bellomo R, Ronco C. Does this patient have acute kidney injury? An AKI checklist. Intensive Care Med 2016; 42 (1): 96-99.

12. Harrois A, Libert N, Duranteau J. Acute kidney injury in trauma patients. Curr Opin Crit Care 2017; 23 (6): 447-456.

13. Hjortrup PB, Haase N, Wetterslev M et al. Clinical review: Predictive value of neutrophil gelatinase-associated lipocalin for acute kidney injury in intensive care patients. Crit Care 2013; 17 (2): 211.

14. Peters H, Macke C, Mommsen P et al. Predictive Value of Osteoprotegerin and Neutrophil Gelatinase-associated Lipocalin on Multiple Organ Failure in Multiple Trauma. In Vivo 2019; 33 (5): 1573-1580. DOI: 10.21873/invivo.11639

15. Rashidi HH, Sen S, Palmieri TL, Blackmon T, Wajda J, Tran NK. Early Recognition of Burn- and Trauma-Related Acute Kidney Injury: A Pilot Comparison of Machine Learning Techniques. Sci Rep 2020; 10 (1): 205. DOI: $10.1038 / \mathrm{s} 41598-019-57083-6$.

16. Janak JC, Stewart IJ, Sosnov JA et al. Urinary Biomarkers are associated with Severity and Mechanism of Injury. Shock 2017; 47 (5): 593-598.

17. Stewart IJ, Glass KR, Howard JT et al. The potential utility of urinary biomarkers for risk prediction in combat casualties: a prospective observational cohort study. Crit Care 2015; 19: 252.

18. Klein SJ, Brandtner AK, Lehner GF et al. Biomarkers for prediction of renal replacement therapy in acute kidney injury: a systematic review and meta-analysis. Intens Care Med 2018; 44: 323-336.

19. Pipili C, Ioannidou S, Tripodaki ES et al. Prediction of the renal replacement therapy requirement in mechanically ventilated critically ill patients by combining biomarkers for glomerular filtration and tubular damage. J Crit Care 2014; 29 (4): 692.e7-13.

20. Beitland S, Moen H, Os I. Acute kidney injury with renal replacement therapy in trauma patients. Acta Anaesthesiol Scand 2010; 54: 833-840.

21. Palmer CS, Gabbe BJ, Cameron PA. Defining major trauma using the 2008 Abbreviated Injury Scale. Injury 2016; 47 (1): 109-115.

22. Wutzler S, Maegele M, Wafaisade A et al. Risk stratification in trauma and haemorrhagic shock: scoring systems derived from the TraumaRegister DGU (®). Injury 2014; 45 Suppl 3: S29-34.

23. Skinner DL, Hardcastle TC, Rodseth RN, Muckart DJ. The incidence and outcomes of acute kidney injury amongst patients admitted to a level I trauma unit. Injury 2013; 45 (1): 259-264. 
24. Yadollahi M, Kashkooe A, Rezaiee R, Jamali K, Niakan MH. A Comparative Study of Injury Severity Scales as Predictors of Mortality in Trauma Patients: Which Scale Is the Best? Bull Emerg Trauma 2020; 8 (1): 27-33. DOI: $10.29252 /$ beat-080105

25. Galkova K, Vrabelova M. Normalization of blood lactate as early end-point of polytrauma treatment. Bratisl Med J 2013; 114 (11): 637-641.

26. Beitl E, Banasova A, Vlcek M, Mikova D, Hampl V. Nitric oxide as an indicator for severity of injury in polytrauma. Bratisl Med J 2016; 117 (4): 217-220.

27. Halvachizadeh S, Baradaran L, Cinelli P, Pfeifer R, Sprengel K, Pape HC. How to detect a polytrauma patient at risk of complications: A validation and database analysis of four published scales. PLoS One 2020; 15 (1): e0228082.

28. Glodowski SD, Wagener G. New insights into the mechanisms of acute kidney injury in the intensive care unit. J Clin Anesth 2015; 27 (2): $175-180$.

29. Hauser CJ, Otterbein LE. Danger signals from mitochondrial DAMPS in trauma and post-injury sepsis. Eur J Trauma Emerg Surg 2018; 44 (3): 317-324.

30. Hesselink L, Hoepelman RJ, Spijkerman R et al. Persistent Inflammation, Immunosuppression and Catabolism Syndrome (PICS) after Polytrauma: A Rare Syndrome with Major Consequences. J Clin Med 2020; 9 (1): 191.

31. Bihorac A, Baslanti TO, Cuenca AG et al. Acute kidney injury is associated with early cytokine changes after trauma. J Trauma Acute Care Surg 2013; 74 (4): 1005-1013.

32. Apple CG, Miller ES, Loftus TJ et al. Impact of Injury Severity on the Inflammatory State and Severe Anemia. J Surg Res 2020; 248: 109-116.
33. Qiao Z, Wang W, Yin L et al. Using IL-6 concentrations in the first $24 \mathrm{~h}$ following trauma to predict immunological complications and mortality in trauma patients: a meta-analysis. Eur J Trauma Emerg Surg 2018; 44 (5): 679-687.

34. AlRawahi AN, AlHinai FA, Doig CJ et al. The prognostic value of serum procalcitonin measurements in critically injured patients: a systematic review. Crit Care 2019; 23 (1): 390.

35. Ahmed AI, Soliman RA, Samir S. Cell Free DNA and Procalcitonin as Early Markers of Complications in ICU Patients with Multiple Trauma and Major Surgery. Clin Lab 2016; 62 (12): 2395-2404.

36. Chico-Fernández M, Barea-Mendoza JA, Ormazabal-Zabala T et al. Associated risk factors and outcomes of acute kidney injury in severe trauma: Results from the Spanish trauma ICU registry (RETRAUCI) (published online ahead of print, 2020 Apr 11). Anaesth Crit Care Pain Med 2020; S2352-5568 (20)30062-X.

37. Huerta-Alardín AL, Varon J, Marik PE. Bench-to-bedside review: Rhabdomyolysis - an overview for clinicians. Crit Care 2005; 9: 158-169.

38. Kasaoka S, Todani M, Kaneko T, Kawamura Y, Oda Y, Tsuruta R et al. Peak value of blood myoglobin predicts acute renal failure induced by rhabdomyolysis. J Crit Care 2010; 25 (4): 601-604.

39. Premru V, Kovac J, Ponikvar R. Use of Myoglobin as a Marker and Predictor in Myoglobinuric Acute Kidney Injury. Ther Apheresis Dialysis 2013; 17 (4): 391-395.

40. Vasquez CR, DiSanto T, Reilly JP et al. Relationship of body mass index, serum creatine kinase, and acute kidney injury after severe trauma (published online ahead of print, 2020 Apr 8). J Trauma Acute Care Surg 2020; 10.1097/TA.0000000000002714. 